\title{
SMART INTEGRATION OF ELECTRIC VEHICLES IN AN ENERGY COMMUNITY
}

\author{
Ebisa Negeri and Nico Baken \\ Delft University of Technology, Delft, The Netherlands
}

Keywords: $\quad$ Smart Grid, Load Balancing, Distributed Generation, Electric Vehicle.

Abstract: With increasing penetrations of renewable distributed generations (DGs) and electrified vehicles (EVs), the volatility of the renewable sources and the huge load of the EVs induce tremendous challenges for the power grid. The two technologies also have considerable synergetic potential to alleviate these challenges if they are intelligently coordinated. The aim of this paper is to investigate how the (dis)charging of EVs could be intelligently coordinated with the production of the local DGs to reduce the peak load on the power grid. We consider a neighborhood energy community that is composed of prosumer households. Three EV (dis)charging scenarios are compared: the dumb strategy where all EVs are charged for the next commute as soon as they return from the previous commute, the centralized (dis)charging strategy where the EVs are managed by a centralized scheduling unit, and the distributed (dis)charging strategy where the households autonomously schedule their EVs while coordination is achieved through providing dynamic pricing based incentives. Our simulation results show that the distributed and centralized charging strategies can reduce the peak load up to $44.9 \%$ and $75.1 \%$, respectively, compared to the dumb charging strategy. Moreover, the relative performnce of the algorithms with respect to environmental values.

\section{INTRODUCTION}

As distributed generations (DGs) are increasingly penetrating into the lower parts of the power grid, the end consumers, such as households, are evolving from passive consumers to active prosumers. According to the EU parliament, all buildings built after 2019 will have to produce their own energy on site $^{1}$. Production of own power on site leads to formation of energy communities (Melo and Heinrich, 2011) that could autonomously manage their own resources and locally exchange power with themselves. On the other hand, the increasing trend in electrification of the transportation sector is expected to bring about massive presence of electrified vehicles (EVs) in the near future.

Since most of the DGs have intermittent electricity production patterns, their large composition might lead to unacceptable volatile profiles that disrupt the grid stability, power quality, and infrastructure constraints. Likewise, presence of an EV doubles the average power demand of a household (Ipakchi and

\footnotetext{
${ }^{1}$ European Parliament, "All New Buildings to be Zero Energy from 2019," Committee on Industry, Research and Energy, Brussels 2009.
}

Albuyeh, 2009), thus its massive presence imposes very large load on the grid. On the other hand, the EVs provide considerable flexibility that could be exploited to minimize these challenges. Given that EVs are idle $95 \%$ of the time, more than $90 \%$ of all vehicles are parked at any given point in time with more than $25 \%$ of them parked at home (Fluhr et al., 2010), their charging could be conveniently shifted to periods of surplus local production. Moreover, the vehicle-to-grid (V2G) technology gives the possibility of discharging from the EV back to the grid during peak demand periods. Thus, by coordinating the (dis)charging of the EVs with the local production of DGs, one can reduce the load of the EVs on the grid, shield the intermittence of the DGs from the grid, and reduce the carbon footprint of the EVs. Accordingly, EVs and DGs seem to form a natural combination.

Although there are a few works in the literature that consider the synergy between the renewable sources and the EVs ((Markel et al., 2009), (Lund and Kempton, 2008), (Verzijlbergh et al., 2011)), they focus on a high-level study of the coordination of fleets of EVs and large scale renewable sources on large geographical regions, without considering the local effects on the grid constraints and the driving behaviors of the users of the EVs. In this paper, we endeavor 
to fill this gap by investigating how the (dis)charging of EVs can be efficiently coordinated with the local generation of power from DGs to minimize the peak load while considering the local grid constraints and the driving behaviors. We consider a model futuristic residential neighborhood energy community that is described in the next section. We present three different EV (dis)charging strategies: a reference dull strategy, a centralized strategy and a distributed strategy. The relative gain of these strategies are compared.

The remainder of this paper is organized as follows. The architecture of the energy community is presented in Section 2. After describing the system model in Section 3, we go on to present the three coordination strategies in Section 4. In Section 5, we present and discuss the simulation results, then we finalize the paper with the concluding remarks in Section 6.

\section{THE NEIGHBORHOOD ENERGY COMMUNITY}

In our work, we consider a neighborhood energy community that is connected to a single medium-voltage to low-voltage (MV/LV) transformer. A simplified diagram of such a neighborhood is shown in Figure 1. The energy community comprises of prosumer households. The prosumer households can autonomously manage their own load profile. Each household can generate, store, import or export power. Power can be exchanged between the prosumer households in the community as well as between the energy community and the MV grid in either direction.

A multi-agent based control is used to coordinate the households. Each household has a householdagent that coordinates its resources to optimize the household consumption. A community-agent watches over the overall load distribution of the community and coordinates the household-agents accordingly. In addition, the community-agent manages the energy exchange with the rest of the grid.

The energy community is assumed to have a smart grid infrastructure in place. Each agent in the energy community has an intelligent device that can exchange information with the other agents across a communication infrastructure, and process the information to make decisions.

\section{THE SYSTEM MODEL}

In this section, the mathematical model of the energy

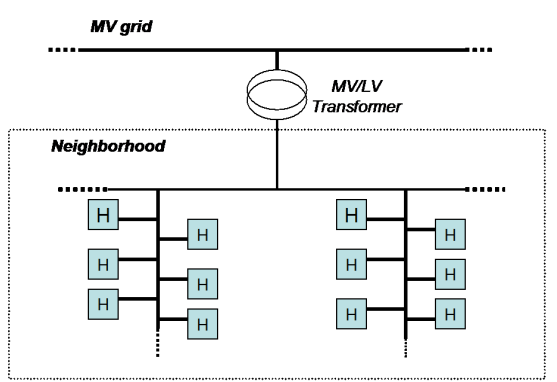

Figure 1: A schematic diagram of a neighborhood energy community.

community is presented. We consider a scheduling time period $\tau(24 \mathrm{~h})$ that is divided into time steps, i.e. $\tau=\{1,2, \ldots, T\}$, that have equal duration of $\Delta$ time units (15 min.).

\subsection{Electric Vehicle}

To characterize each EV, the parameters of its battery are important. The battery parameters of interest for this work are the following: the maximum energy storage capacity $(\Phi$ in $k W h)$, the cycle efficiency $(\eta$ in $\%)$, the maximum depth of safe discharging to prolong battery life ( $\delta^{c h}$ in $\%$ ), and the maximum level of safe charging to prolong battery life ( $\left.\delta^{d c h} \%\right)$. Assuming a symmetric cycle efficiency, we denote the storage charging efficiency and discharging efficiency by $\eta^{c h}$ and $\eta^{d c h}$, respectively, where $\eta^{c h}=\eta^{d c h}=\sqrt{\eta}$. The state of charge (SOC) of the battery at time step $i$ is denoted by $\psi_{i}$. The amount of electrical power consumed to charge the battery of the EV, and the amount of electrical power supplied from the battery upon discharging (V2G) in time step $i$ are denoted by $X_{i}$ and $Y_{i}$, respectively. After the EV commutes a distance of $l \mathrm{~km}$, the state of charge of its battery drops by an amount $l \times \omega$, where $\omega$ is the average amount of stored battery energy the EV consumes per unit km travelled (in $\mathrm{kWh} / \mathrm{km}$ ).

In this work, we focus on the impact of EVs on the power grid of a residential energy community. Thus, we consider only the case where the EVs are charged and discharged (V2G) within the energy community either at home or at a charging station within the community. We denote the periods of time the EV stays within its community and away by $\tau^{h}$ and $\tau^{-h}$, respectively. The usage of EV is subject to the constraints listed in Eq.1-8. Eq.1 states that the SOC at the end of a time step $j$ can be obtained from the SOC at the end of the previous time step $i$ by adding the net rise in the SOC in time step $j$. The net rise in the SOC results from the injected energy via charging $\left(\Delta \eta^{c h} X_{j}\right)$, the supplied energy via discharging $\left(\Delta \eta^{d c h} Y_{j}\right)$ and the 
battery energy used in commuting $\left(\pi_{j}\right) . \Delta$ is employed here to convert power into energy. Constraints 2 and 3 represent the maximum and minimum boundaries, respectively, on the SOC for safety of the battery. Constraint 4 says that the battery of the EV cannot be charging and discharging at the same time. On the other hand, constraints 6 and 5 enforce the maximum and minimum charging and discharging rates of the battery, respectively, where $v^{c h}$ and $v^{d c h}$ denote the maximum charging and discharging rates, respectively, of the charger. Constraint 7 reflects that we are limiting the (dis)charging of the EVs only to the periods when they are within their community. Whenever an EV begins to commute a distance of $l \mathrm{~km}$, its battery level should be large enough to commute the distance, and yet not fall below the minimum safe SOC after commuting (constraint 8).

The deriving behavior of the EV user is derived from a mobility data, as will be described in section 5 .

$$
\begin{aligned}
\psi_{j} & =\psi_{i}+\Delta\left(\eta^{c h} X_{j}-\eta^{d c h} Y_{j}\right)-\pi_{j}, \forall i, j \in \tau \\
\psi_{i} & \leq \delta^{c h} \Phi, \forall i \in \tau \\
\psi_{i} & \geq \delta^{d c h} \Phi, \forall i \in \tau \\
X_{i} & =0 \text { or } Y_{i}=0, \forall i \in \tau \\
0 & \leq Y_{i} \leq v^{d c h}, \forall i \in \tau \\
0 & \leq X_{i} \leq v^{c h}, \forall i \in \tau \\
X_{i}, Y_{i} & =0, \forall i \in \tau^{-h} \\
\psi_{i} & \geq \delta^{d c h} \Phi+l w, \text { commute of } l \mathrm{~km} \text { begins at } i
\end{aligned}
$$

\subsection{Household Demand and Production}

The accumulated power demand of all the appliances of the household in time step $i$ is denoted by $D_{i}$. This demand does not include the demand of the EV owned by the household, because the EV is modeled separately. We designate the total power production of all the DGs owned by the household in time step $i$ by $P_{i}$. In this work, we assume that forecasts of $D$ and $P$ are available for the entire scheduling period. The net demand of a household in time step $i$, denoted by $R_{i}$, is defined as the difference between the demand (appliances and EV) and the production of the household (Eq.9). $R_{i}$ is upper bounded by the power capacity of the line connecting the household to the grid, $L^{\text {th }}$ (Eq.10).

$$
\begin{aligned}
& R_{i}=\left[D_{i}+\left(X_{i}-Y_{i}\right)\right]-P_{i}, \forall i \in \tau \\
& R_{i} \leq L^{t h}, \forall i \in \tau
\end{aligned}
$$

\subsection{Transformer}

The net power flow through the transformer, that con- nects the community to the rest of the grid, at any time step $i$ is equal to the net demand of the community, $P_{i}^{\text {net }}$ given in Eq.11, and is generally constrained by the threshold capacity of the transformer $\left(P^{t h}\right)$, as shown in Eq.12. In our case, we loosen the constraint in Eq.12 and try to minimize the peak load using different charging strategies. Observing the resulting peak load helps to suggest the optimal value of the transformer rating to be installed to support the load profile in the energy community under consideration.

$$
\begin{aligned}
& P_{i}^{\text {net }}=\left(\sum_{\text {all houses }} R_{i}\right), \forall i \in \tau \\
& P_{i}^{\text {net }} \leq P^{\text {th }}, \forall i \in \tau
\end{aligned}
$$

\section{THE CHARGING STRATEGIES}

In this section, we present three EV charging strategies, namely, the dull strategy, the centralized strategy, and the distributed strategy.

\subsection{The Dull Strategy}

This strategy represents a reference scenario where the EVs are charged for their next commute as soon as they return to the community from their previous commute. The EVs are charged up to the target battery level without interruption at the maximum possible rate. Apparently, this strategy does not possess any intelligence, hence the name dumb.

\subsection{The Centralized Strategy}

The centralized charging strategy assumes that the energy community is composed of prosumers that cooperate with each other to achieve communal goals. In this case, (dis)charging of the EVs are scheduled offline in a centralized way by an intelligent scheduling unit (ISU) that could be located at the communityagent. As opposed to the dumb strategy, this strategy allows charging of the EVs with variable charging rates and with possible interruptions. This strategy finds optimal schedules as a solution to a linear programming problem that is shown in Eq.13, where peak denotes the peak value of the net demand of the energy community $\left(P^{n e t}\right)$. Apparently, this strategy aims at aligning the EVs with the local production to minimize the peak load on the transformer.

$$
\begin{aligned}
& \text { minimize peak } \\
& \text { subject to Eq. 1-12 }
\end{aligned}
$$




\subsection{The Distributed Strategy}

This strategy is based on the assumption that each household tends to selfishly maximize its own benefits. The EVs are autonomously scheduled by their respective owners. Thus, a coordination mechanism is required to maximize utilization of the locally generated power. Our coordination mechanism involves a local electronic energy market (Kok et al., 2005) within the community where the autonomous households trade electricity with each other, whereby the autonomy of the energy community allows the community-agent to propose local price-vectors to achieve desirable communal goals. The communityagent provides incentives based on a novel dynamic pricing model that aims at motivating the community members to shift their demands to lower the peak load on the transformer.

\subsubsection{The Cost}

To handle the power import and export of each household, the dynamic pricing model has two components for each time step $i$ : the import tariff $\left(\gamma_{i}^{i m}\right)$ and the export tariff $\left(\gamma_{i}^{e x}\right)$. The cost of the household is modeled in Equation 14 for each time step $i$, where the terms $\left(\gamma_{i}^{i m} * R_{i}\right)$ and $\left(\gamma_{i}^{e x} * R_{i}\right)$ represent the monetary cost and benefit, respectively, incurred due to the net demand of the household.

$$
C_{i}^{h}=\left\{\begin{array}{l}
\gamma_{i}^{i m} * R_{i}, \text { if } R_{i} \geq 0 \\
\gamma_{i}^{e x} * R_{i}, \text { if } R_{i}<0
\end{array}\right.
$$

\subsubsection{The Household Optimization}

Each household schedules its EVs to minimize its cost by solving a linear household optimization problem that is shown in Equation 15.

$$
\text { minimize } \sum_{\forall i \in \tau} C_{i}^{h}
$$

subject to Equations 1-9, 14.

\subsubsection{The Dynamic Pricing Model}

In order to flatten the overall load distribution of the neighborhood, the community-agent provides each household with an incentive that is based on a dynamic pricing model. In our dynamic pricing model, the community-agent proposes a price vector, then each household-agent schedules the storage unit of that household and replies with the corresponding scheduled demand of the household. Based on the response of all the households, the community-agent adjusts the price vector using an intelligent learning mechanism. This is repeated iteratively to obtain a flattened profile.

In the proposed pricing model, the cost of a unit power varies from one time step to another depending on the net demand of the neighborhood energy community $\left(P^{n e t}\right)$. Our pricing model aims at encouraging the households to shift their scheduled demands away from the peak periods of the overall scheduled demand of the neighborhood. The optimal tariff for each time step is determined iteratively. At the $(j+1)^{\text {th }}$ iteration cycle, the tariff for the $i^{t h}$ time step is obtained by adjusting the corresponding tariff in the previous iteration cycle $j$ using an incremental factor $\left(\gamma \times \xi_{j, i}\right)$ :

$$
\theta_{j+1, i}=\theta_{j, i}\left(1+\gamma \times \xi_{j, i}\right)
$$

The incremental factor is composed of two terms: the learning factor $(\gamma)$ and the deviation factor $\left(\xi_{j, i}\right)$. The learning factor is a constant $(0<\gamma \leq 1)$ that determines to which extent the deviation factor overrides the old price vector. The deviation factor captures the variation of $P^{\text {net }}$ from its average value. Let $P_{i}^{\text {net }}$ be the net demand of the energy community in the $i^{t h}$ time step for the $j^{\text {th }}$ iteration cycle. Let mean be the average of the net demand:

$$
\text { mean }=\frac{1}{T} \sum_{i=1}^{T} P_{i}^{n e t}
$$

The deviation factor, $\xi_{j, i}$, is given by

$$
\xi_{j, i}=\operatorname{sign} \times \frac{\left(P_{i}^{n e t}-m e a n\right)^{2}}{\sum_{i=1}^{T}\left(P_{i}^{n e t}-m e a n\right)^{2}}
$$

where the sign determines whether the incremental value should be positive or negative. If $P_{i}^{n e t}>$ mean, then we add a positive incremental value to the tariff to reduce consumption in this time step: $\operatorname{sign}=1$. If $P_{i}^{\text {net }}<$ mean, then we substract an incremental value from the tariff to increase consumption in this time step: sign $=-1$. Clearly, $\xi_{j, i}$ reflects the effect of the offset of the demand from the mean value on the price vector. The pricing model increases tariff on the time steps where the overall scheduled demand of the neighborhood community $\left(P^{\text {net }}\right)$ is above the average, and reduces tariff when $P^{n e t}$ is below the average, thereby providing incentives to the households to flatten the overall scheduled neighborhood demand.

The power feed-in tariffs are obtained by substracting the transport part of the import tariffs as suggested in (Houwing et al., 2011). Consequently,

$$
\lambda_{j}=\theta_{j}-\theta^{t r}, \forall j \in \tau
$$

where $\theta^{t r}$ is the transport part of the import tariff $\theta_{j}$.

\subsubsection{The Distributed Algorithm}

Our proposed distributed algorithm comprises of two types of algorithms. The first type of algorithm (Algorithm 1) is implemented at the community-agent 
while the other (Algorithm 2) is implemented at each household-agent. These algorithms iteratively solve the household optimization problems by exchanging information. The iteration cycles continue for a fixed number of iterations maxIteration, while searching for an optimal price vector that yields the smallest peak value for the overall net demand of the neighborhood energy community $\left(P^{n e t}\right)$.

The block diagram describing the execution of the algorithms is shown in Figure 2. At each iteration step, the community-agent sends the recent tariff information to each household-agent. Then, it receives the scheduled demand of the households subject to the corresponding tariff. Afterwards, it computes the tariffs for the next iteration cycle based on the overall scheduled demand of the community $\left(P^{n e t}\right)$. On the other hand, each household-agent in each iteration cycle solves its household optimization problem using the tariff information received from the communityagent. It then sends the updated scheduled demand of the household $(R)$ to the community-agent.

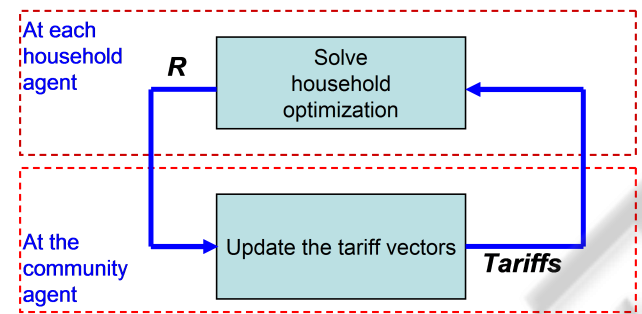

Figure 2: Block diagram of the distributed charging strategy.
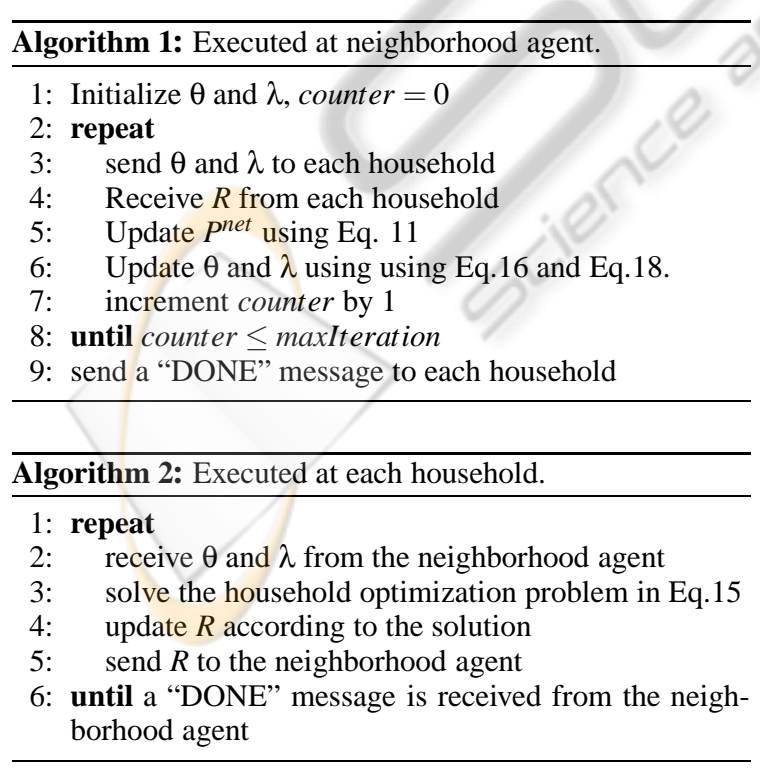

Solving the optimization problems is initiated at the community-agent (Algorithm 1). The algorithm starts by initializing the tariffs ( $\theta$ and $\lambda$ ) to flat price vectors and the iteration cycle counter (counter) to zero in line 1. In each iteration cycle, lines 3-7 are executed. In line 3 , the recent tariff information is sent to each household-agent which will be used by the household-agent to solve the household optimization problem in the current iteration cycle. It then receives from each household-agent its scheduled demand $(R)$ resulting from the solution of the household optimization problem in the current iteration cycle (line 4). After computing the overall net demand of the neighborhood $P^{n e t}$ in line 5, the tariffs used in the next iteration cycle are updated accordingly (line $6)$. The iteration continues until the iteration count is equal to maxIteration. The algorithm finalizes the optimization process by sending the "DONE" message to all the household-agents (line 9). The optimal tariff is chosen by selecting tariff vectors that yielded the lowest peak value of $P^{\text {net }}$.

The algorithm at each household-agent (2) iteratively solves the household optimization problem. In each iteration cycle, the algorithm receives the upto-date tariff information from the community-agent (line 2), solves its optimization problem using the new tariffs (line 3), updates the scheduled demand of the household according to the solution (line 4) and sends it back to the neighborhood-agent (line 5). The iteration continues until a "DONE" message is received.

\section{SIMULATION}

\subsection{Simulation Data}

The data for the demand and electricity production of a household are acquired from Alliander ${ }^{2}$. The DGs of the household are a PV panel and a microCHP. The data were obtained by field measurements that were taken every 15 minutes for a duration of 24 hours using smart meters installed at the households. The micro-CHP generation is constant $(1 \mathrm{~kW})$ over each period of operation, and the data specifies the start time and end time of each period of its operation. To make alternative profiles for each household, we randomized the values according to a normal distribution around the measured values. A sample of the daily production and demand profile of a household is shown in Fig. 3.

The driving behavior of the EV users are constructed based on the data from the 2009 mobility re-

\footnotetext{
${ }^{2}$ Alliander is the largest electricity distribution network operator company in the Netherlands owning $40 \%$ of the distribution networks.
} 


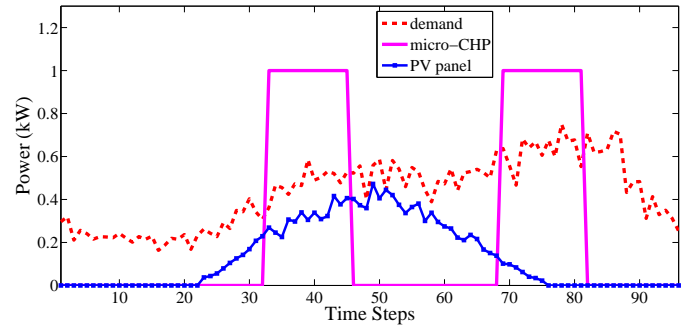

Figure 3: A sample household demand and production profiles.

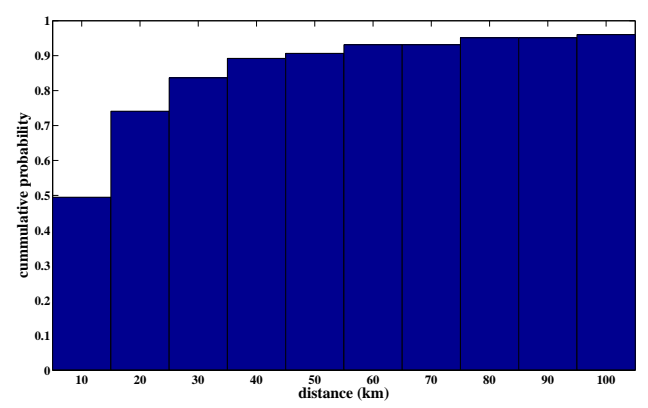

Figure 4: Distribution of the distance travelled per day per car driver.

search of the Netherlands (MON, 2009). Fig. 4 shows the distribution of the distance traveled per car driver per day as extracted from the data. The commute distances used in the simulations are generated using a function with exponential distribution that best fits the data. For each commute, the departure and arrival time from/at home are generated using Gaussian functions that best approximate the data obtained from (MON, 2009).

In our simulations, we involve three types of electric cars whose technical specifications and market share are specified in (Knapen et al., 2011), as presented in Table 1. The composition of the EVs in the energy community is in line with the market share values in Table 1 . The values of the cycle efficiency of the batteries, the safe charging level and the safe discharging level are adopted from (Lombardi et al., $2009)$, with the values $\eta=95 \%, \delta^{\text {ch }}=90 \%$, and $\delta^{d c h}=30 \%$. We conducted our simulations with community size, $N=50$ households, with a conservative assumption that each household owns one EV. The average number of cars per household is about 1.6 according to (MON, 2009), thus our assumption of one EV per household represents roughly $62 \%$ penetration of the EVs. We assume that each household is connected to the grid with a standard connection of $230 \mathrm{~V}$ and $40 \mathrm{~A}$ (the case in The Netherlands) resulting in a line limit of $L^{\text {th }}=9.2 \mathrm{~kW}$.
Table 1: Technical characteristics for vehicles in specific categories, and their market share (Knapen et al., 2011).

\begin{tabular}{|c|c|c|c|}
\hline EV category & small & medium & large \\
\hline Market share & 0.496 & 0.364 & 0.140 \\
\hline$\Phi[\mathrm{kWh}]$ & 10 & 20 & 35 \\
\hline Range $[\mathrm{km}]$ & 100 & 130 & 180 \\
\hline$\omega[\mathrm{kWh} / \mathrm{km}]$ & 0.1 & 0.15 & 0.19 \\
\hline
\end{tabular}

\subsection{Simulation Results}

We present different cases that compose our simulation scenarios. We consider two types of chargers: slow charger $\left(v^{\text {ch }}=3.3 \mathrm{KVA}\right)$ and fast charger $\left(v^{c h}=7.2 \mathrm{kVA}\right)$, that are compatible with the Flemish grid (Knapen et al., 2011). Moreover, we consider two cases: charging with V2G and without V2G. While the first case allows feeding from the EV battery back to the grid, the later does not allow it. We assume that the rate of $\mathrm{V} 2 \mathrm{G}$ discharging is constrained by the same threshold value, i.e., $v^{d c h}=v^{c h}$. In addition, two cases are considered concerning the target battery level at the start time of each commute. In the first case we assume that the target battery level is just sufficient to support the commute (Eq. 8), whereas in the second case the target battery level is the maximum safe charging level of the battery ( $90 \%$ of $\Phi)$. Combining these cases, we present eight simulation scenarios to compare the performance of our charging strategies.

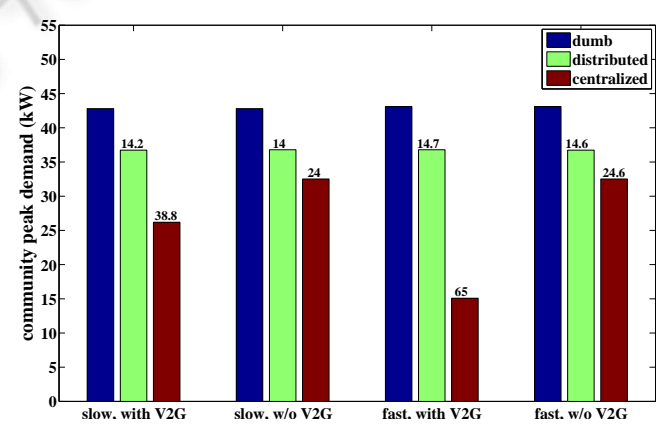

Figure 5: Comparison of the algorithms based on the peak demand of the community (target battery level is commute energy).

For the case when the target battery level at the start of each commute is the energy required for the commute, the relative performance of the charging strategies under four different scenarios are presented in Fig.5. The numbers on the top of the bars representing the distributed and the centralized charging strategies denote the percentage reduction in the peak load as compared to the dumb charging strategy. As can be observed from the figure, the distributed charging 


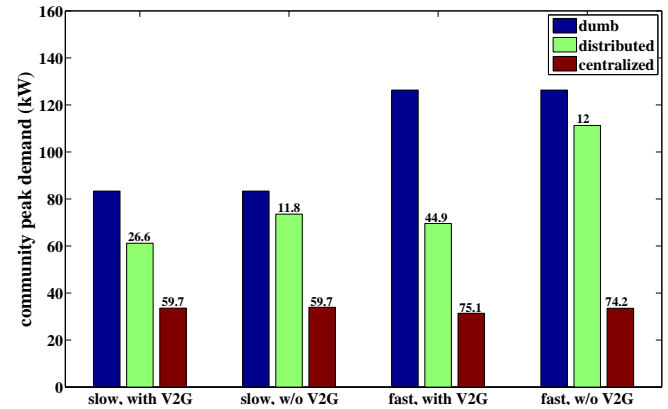

Figure 6: Comparison of the charging strategies based on the peak demand of the community (target battery level is $90 \%$ of the battery capacity).

strategy yields a reduction in the peak load by around $14 \%$ in all the four scenarios, which average to $14.4 \%$. On the other hand, the centralized charging strategy reduces the peak by as large as $65 \%$, with average improvement of $38.1 \%$.

Figure 6 depicts the relative performance of the charging strategies when the target battery level at the start of each commute is $90 \%$ of the battery capacity. In this scenario, the distributed charging strategy achieves an average peak reduction of $23.9 \%$, where its largest reduction is as high as $44.9 \%$. Whereas the centralized charging strategy obtains $67.2 \%$, with $75.1 \%$ largest improvement.

All the simulation results reveal that the dumb charging strategy results in large peak demands which arise from the simultaneous charging of EVs right after they arrive at home. In the contrary, the centralized charging strategy has effectively revealed the potential of the synergy between the EVs and the local production to reduce the peak load by exploiting the flexibility of the EVs. However, the centralized charging strategy represents a situation where all the households are cooperative to reduce the peak load and all the EVs are managed by a centralized unit, which might contradict the tendency of the households to autonomously manage their own EVs. On the other hand, the distributed charging strategy delivers $11 \%$ to $44.9 \%$ reduction in peak demand over the dumb charging strategy while respecting the autonomy of the households to manage their own EVs. The strength of the distributed strategy lies in the dynamic pricing model that is used as incentive to coordinate the autonomous households that tend to selfishly minimize their cost. These improvements are vital because they reduce the load on the transformer connecting the energy community with the remaining power grid. The reduction in the peak values has the advantage of increasing the lifetime of the transformer as well as minimizing the need for installing large capacity transformer.

\section{CONCLUSIONS}

In an energy community composed of prosumer households that own EV, the aggregate load profile might become highly volatile due to the intermittence of the distributed generations as well the load of the EVs, which could lead to a large peak load that might exceed the capacity of the transformer. We have proposed a centralized and a distributed EV charging strategies that try to minimize the peak load in such scenario by exploiting the synergy between the local distributed generations and the flexibility of the EVs. Our centralized charging strategy schedules the (dis)charging of the EVs at a centralized unit assuming that all the prosumers in the energy community cooperate with each other to minimize the peak demand. Our distributed charging strategy, however, assumes that the prosumer households autonomously manage their EVs and tend to selfishly minimize their cost. Our distributed strategy coordinates the households to minimize the peak demand by providing incentives based on our novel dynamic pricing model. In addition to presenting a detailed model of the system taking into consideration the local constraints, we have derived the driving behaviors of the EV users from a realistic mobility data. Based on our simulation results, we have shown that our centralized and distributed charging strategies can reduce the peak by as large as $75.1 \%$ and $44.9 \%$, respectively, compared to a reference dumb charging strategy. Therefore, our proposed strategies can help to reduce the load on the transformer connecting the energy community to the rest of the grid, and also minimizes the need for upgrading the capacity of the transformer.

\section{REFERENCES}

Fluhr, J., Ahlert, K., and Weinhardt, C. (2010). A stochastic model for simulating the availability of electric vehicles for services to the power grid. In 43rd HICSS, 2010, pages 1-10. IEEE.

Houwing, M., Negenborn, R., and De Schutter, B. (2011). Demand response with micro-chp systems. Proceedings of the IEEE, 99(1):200-213.

Ipakchi, A. and Albuyeh, F. (2009). Grid of the future. Power and Energy Magazine, IEEE, 7(2):52-62.

Knapen, L., Kochan, B., Bellemans, T., Janssens, D., and Wets, G. (2011). Activity based models for countrywide electric vehicle power demand calculation. $S G M S$.

Kok, J., Warmer, C., and Kamphuis, I. (2005). Powermatcher: multiagent control in the electricity infrastructure. In Proceedings of the fourth international joint conference on Autonomous agents and multiagent systems, pages 75-82. ACM. 
Lombardi, P., Vasquez, P., and Styczynski, Z. (2009). Plugin electric vehicles as storage devices within an autonomous power system. optimization issue. In PowerTech, 2009 IEEE Bucharest, pages 1-7. IEEE.

Lund, H. and Kempton, W. (2008). Integration of renewable energy into the transport and electricity sectors through v2g. Energy Policy, 36(9):3578-3587.

Markel, T., Kuss, M., and Denholm, P. (2009). Communication and control of electric drive vehicles supporting renewables. In $V P P C^{\prime} 09$. IEEE, pages 27-34. IEEE.

Melo, H. and Heinrich, C. (2011). Energy balance in a renewable energy community. IEEE EEEIC.

MON (2009). Mobility research of the netherlands 2009 (mobilitietsonderzoek nederland (mon) 2009). available: http://rijkswaterstaat.nl/.

Verzijlbergh, R., Ilic, M., and Lukszo, Z. (2011). The role of electric vehicles on a green island. In NAPS, 2011, pages $1-7$. IEEE. 